\title{
Positive Culture Prior to Transperineal Prostate Biopsy Was Not Associated with Post-Biopsy Febrile Urinary Tract Infection Development
}

\section{Yoichiro Tohi (1) \\ Kengo Fujiwara \\ Satoshi Harada \\ lori Matsuda \\ Ayako Ito \\ Mari Yamasaki \\ Yasuyuki Miyauchi \\ Yuki Matsuoka \\ Takuma Kato \\ Rikiya Taoka \\ Hiroyuki Tsunemori \\ Nobufumi Ueda \\ Mikio Sugimoto}

Department of Urology, Faculty of Medicine, Kagawa University, Kagawa, Japan
Purpose: To investigate the association between urine culture before transperineal prostate biopsy and post-biopsy febrile urinary tract infection (fUTI).

Patients and Methods: We retrospectively reviewed 307 patients who underwent urine culture before transperineal prostate biopsy between April 2017 and September 2020. Patients with indwelling urinary catheters $(n=7)$ were excluded. Urine culture was performed 1-3 days before the biopsy, and all patients received prophylactic cefazolin regardless of culture results. A urine culture was defined as positive if cell density was more than $1 \times 10^{5}$ colony-forming units per $\mathrm{mL}$. Baseline characteristics and the incidence of post-biopsy fUTI were compared between patients showing positive pre-biopsy culture results and those showing negative findings.

Results: Out of 300 , seven patients $(2.3 \%)$ had positive urine culture results before the biopsy. Age $(\mathrm{p}=0.077)$; prostate-specific antigen at diagnosis $(\mathrm{p}=0.267)$; prostate volume $(\mathrm{p}=0.78)$; number of biopsy cores $(\mathrm{p}=0.277)$; percentage of patients testing positive for cancer on biopsy $(\mathrm{p}=0.71)$; and percentages of patients with a history of biopsy ( $\mathrm{p}>0.999)$, diabetes mellitus $(p=0.604)$, and immunosuppressive medication use $(p>0.999)$ were similar between the two groups. No patient in the positive urine culture group had post-biopsy fUTI. However, $1.7 \%$ (five patients) of the negative urine culture group had the disease ( $>0.999)$ (four patients with prostatitis and one with pyelonephritis). Among them, two patients were diagnosed by urine culture at the time of post-biopsy fUTI.

Conclusion: In asymptomatic patients, positive pre-biopsy cultures were not associated with the development of post-biopsy fUTI.

Keywords: urinary tract infection, prostate biopsy, transperineal, urine culture

\section{Introduction}

Prostate biopsy (PB) is necessary for diagnosing prostate cancer. Although the efficacy to detect malignancy is similar in both transrectal and transperineal approaches, ${ }^{1}$ many institutions have adopted the transrectal prostate biopsy (TR$\mathrm{PB})$ because of its simplicity, particularly concerning anesthesia. ${ }^{2-4}$

However, TR-PB is associated with a higher incidence of infection than a transperineal prostate biopsy (TP-PB). ${ }^{5}$ With the increasing antibiotic resistance of the rectal flora, ${ }^{6}$ TP-PB should be considered to reduce the risk of infectious complications as it does not involve any passage through the rectum. However, while the frequency of infections in TP-PB is reported to be $0.38-3.82 \%{ }^{7-9}$ these infections could sometimes be severe and even life-threatening. Although some
Correspondence: Yoichiro Tohi Department of Urology, Faculty of Medicine, Kagawa University, Kagawa, I750-I, Ikenobe, Miki-cho, Kita-gun, Kagawa, 76I-0793, Japan

Tel +8I-87-898-2202

Fax $+81-87-898-2203$

Email yoto7I6yotoyoto@gmail.com 
reports suggest that antibiotic prophylaxis is not necessary for TP-PB, ${ }^{10}$ prophylactic therapy is generally recommended to reduce the risk of infection.

Because of the presence of bacteria in feces or urine, consideration of pre-biopsy rectal swab culture is shown to reduce the risk of post-biopsy infection in TR-PB. ${ }^{11}$ Although the presence of bacteria on the perineal skin or in urine can also lead to post-biopsy infections in TP-PB, the clinical utility of pre-biopsy urine culture is not well known.

Here, we aimed to investigate the association between urine culture before transperineal PB and post-biopsy febrile urinary tract infection (fUTI).

\section{Materials and Methods}

This study was approved by our institutional review board (Admission number: 2020-125). The need for informed consent was waived given the retrospective nature of the study. However, information regarding this study was still disclosed on the website, and opportunities for refusal were guaranteed.

\section{Study Design and Patient Population}

We performed a retrospective analysis of 307 patients who underwent urine culture tests before TP-PB between April 2017 and September 2020. Patients were excluded if a urinary catheter was placed.

\section{Protocol}

All patients were hospitalized. Urine culture tests were performed 1-3 days before TP-PB. The urine culture was considered positive if the cell density of more than $1 \times 10^{5}$ colony-forming units per $\mathrm{mL}(\mathrm{CFU} / \mathrm{mL})$ was observed. All patients were treated with prophylactic cefazolin 1 $\mathrm{g}$ intravenously before biopsy regardless of the urine culture results. TP-PB was performed under general or subarachnoid anesthesia of the spine. Perineal and perianal sterilization was performed using iodine, but not in the rectum. A urinary catheter was placed after biopsy. Patients who were stable on the first postoperative day had their urinary catheter removed and were discharged.

\section{Data Collection and Statistical Analysis}

All data were collected from electronic medical records. In this study, baseline characteristics data (age, prostatespecific antigen (PSA) at diagnosis, prostate volume, number of biopsy cores, number of cancer-positive patients identified upon biopsy, and history of biopsy, diabetes mellitus, and immunosuppressive medication use) were collected retrospectively. According to pre-biopsy urine culture results, patients were divided into two groups: (1) positive urine culture group and (2) negative urine culture group. To compare the baseline characteristics between the two groups, chi-square and Fisher's exact tests were used for categorical variables, while the Mann-Whitney $U$-test was used to evaluate continuous variables.

Among patients with positive urine culture results, the types of bacteria isolated were also examined. The incidence of post-biopsy complications (fUTI, dysuria, macrohematuria, and others) within 30 days after biopsy was compared between the two groups. fUTI was defined as a symptomatic status in the genitourinary tract with fever (temperature $>38^{\circ} \mathrm{C}$ ). To diagnose whether or not the patient had pyelonephritis, prostatitis, or epididymitis, we referred to clinical symptoms such as costovertebral angle tenderness, tenderness on rectal examination, and scrotal pain.

Antimicrobial susceptibility was defined according to the Clinical and Laboratory Standards Institute guidelines. A p-value $<0.05$ was considered to be statistically significant. All statistical analyses were performed using IBM SPSS Statistics for Windows, version 25.0 (IBM Corp., Armonk, NY, USA).

\section{Results}

A total of 307 patients who underwent urine culture tests before TP-PB within the study period were initially included. However, patients who had indwelling urethral catheters $(n=7)$ were excluded. Finally, the 300 patients who met the inclusion criteria were selected for this study.

Table 1 shows patient characteristics. Of the 300 patients, positive pre-biopsy urine cultures were identified in seven patients $(2.3 \%)$. Statistical analysis revealed no significant difference between the positive urine culture group and the negative urine culture group in terms of median age ( 74 vs 71 years old, $\mathrm{p}=0.077$ ), median PSA level at diagnosis ( 8.63 vs $6.72 \mathrm{ng} / \mathrm{mL}, \mathrm{p}=0.267)$, median prostate volume ( 30.5 vs $33.2 \mathrm{cc}, \mathrm{p}=0.78$ ), number of biopsy cores ( 14 vs 14 cores, $\mathrm{p}=0.277$ ), percentage of cancer-positive patients identified upon biopsy $(57.1 \%$ vs $63.4 \%, \mathrm{p}=0.71$ ), and percentages of patients with a history of biopsy ( $28.5 \%$ vs $29.3 \%$, p $>0.999)$, diabetes mellitus ( $0 \%$ vs $16.7 \%, \mathrm{p}=0.604)$, and immunosuppressive medication use ( $0 \%$ vs $2 \%, \mathrm{p}>0.999)$.

Figure 1 shows the bacterial characteristics of prebiopsy urine culture. In 12 out of the 300 patients, bacteria 
Table I Patients Characteristics of the 300 Patients Who Were Performed Transperineal Prostate Biopsy

\begin{tabular}{|l|l|l|l|l|}
\hline Variables & All & Positive Urine Culture & Negative Urine Culture & p-value \\
\hline Number of patients, $\mathrm{n}(\%)$ & 300 & $7(2.3)$ & $293(97.7)$ & $74(67-75)$ \\
\hline Median age, years (IQR) & $71(67-76)$ & $74(74-78)$ & $6.72(5.12-10.3)$ & 0.077 \\
\hline Median PSA at biopsy, ng/mL (IQR) & $6.8 I(5.12-10.32)$ & $8.63(6.93-11.75)$ & $33.2(25-48)$ & 0.267 \\
\hline Median prostate volume, cc (IQR) & $33(25-48)$ & $30.5(23-59.5)$ & $14(14-16)$ & 0.78 \\
\hline Median number of biopsy cores, n (IQR) & $14(14-16)$ & $14(12-15)$ & $186(63.4)$ & 0.277 \\
\hline Positive biopsy, n (\%) & $190(63.3)$ & $4(57.1)$ & $86(29.3)$ & 0.71 \\
\hline Prior biopsy, $\mathrm{n}(\%)$ & $88(29.3)$ & $2(28.5)$ & $49(16.7)$ & $0.999<$ \\
\hline Diabetes mellitus, $\mathrm{n}(\%)$ & $49(16.3)$ & $0(0)$ & $6(2)$ & 0.604 \\
\hline Immunosuppression, $\mathrm{n}(\%)$ & $6(2)$ & $0(0)$ & & $0.999<$ \\
\hline
\end{tabular}

Abbreviation: IQR, interquartile range.

were isolated in pre-biopsy urine culture; however, only seven patients had a positive urine culture (ie, $>10^{5} \mathrm{CFU} /$ $\mathrm{mL}$ ). Among these seven patients, five patients had one type of bacteria isolated, and two patients had two types of bacteria isolated in their urine cultures. One of the patients with two types of bacteria isolated had one bacteria with counts $>10^{5} \mathrm{CFU} / \mathrm{mL}$ and one with counts $<10^{5} \mathrm{CFU} / \mathrm{mL}$. The distributions of patients with bacterial species at counts $>10^{5} \mathrm{CFU} / \mathrm{mL}$ in pre-biopsy culture are as follows: Staphylococcus epidermidis ( $\mathrm{n}=3)$, Streptococcus agalactiae $(\mathrm{n}=1)$, Staphylococcus aureus $(\mathrm{n}=1)$, Enterococcus faecalis $(\mathrm{n}=1)$, Staphylococcus caprae $(\mathrm{n}=1)$, and
Raoultella ornithinolytica $(\mathrm{n}=1)$. All other bacteria other than Enterococcus faecalis and Raoultella ornithinolytica were susceptible to cefazolin. Table 2 shows the antimicrobial susceptibility of each bacteria (more than $1 \times 10^{5}$ $\mathrm{CFU} / \mathrm{mL}$ ) on pre-biopsy positive urine culture. Out of seven bacteria available for evaluation, six were susceptible to CEZ (85.7\%). Out of eight bacteria available for evaluation, five were susceptible to LVFX (62.5\%).

Table 3 shows infectious and non-infectious complications following TP-PB, stratified by pre-biopsy urine culture results. There were no cases of post-biopsy fUTI in the positive urine culture group; however, 1.7\% (five

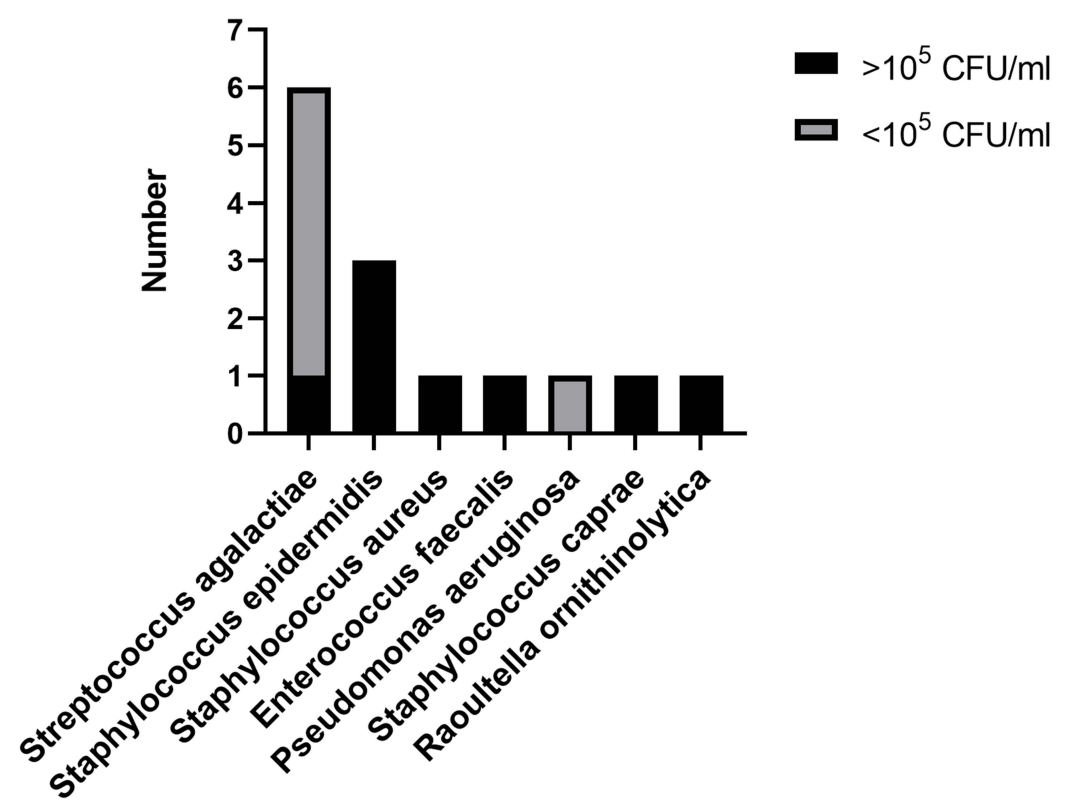

Figure I Bacterial characteristics observed in pre-biopsy urine culture. 


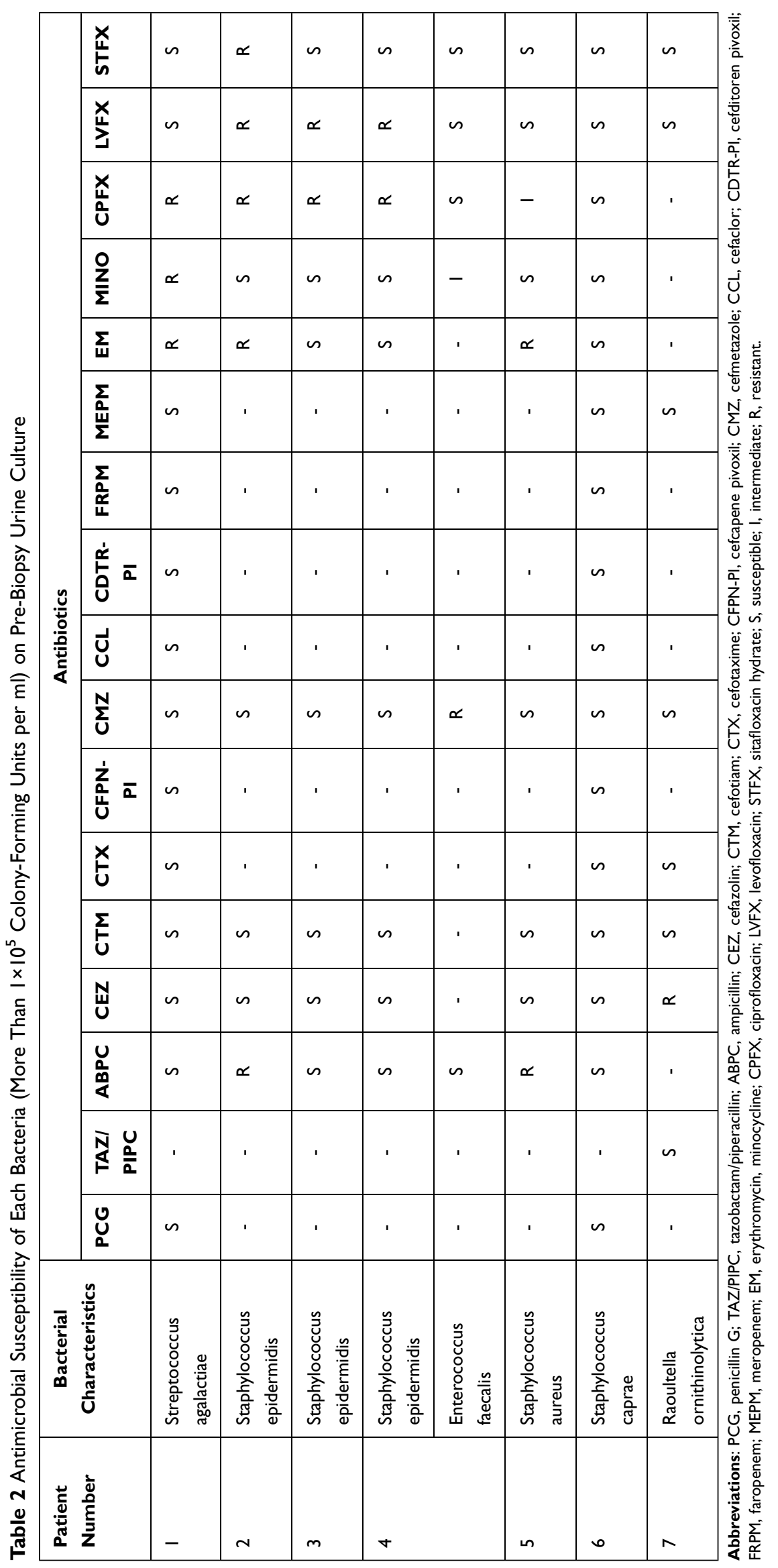


Table 3 Infectious and Noninfectious Complications Following Transperineal Prostate Biopsy

\begin{tabular}{|l|l|l|l|}
\hline Variables & Positive Urine Culture & Negative Urine Culture & p-value \\
\hline Number of patients, $\mathrm{n}$ & 7 & 293 & \\
\hline Any complications, $\mathrm{n}(\%)$ & $3(42.8)$ & $58(19.8)$ & 0.151 \\
\hline Infection, $\mathrm{n}(\%)$ & $0(0)$ & $5(1.7)$ & $0.999<$ \\
$\quad$ Prostatitis, $\mathrm{n}(\%)$ & $0(0)$ & $4(1.4)$ & $1(0.3)$ \\
Pyelonephritis, $\mathrm{n}(\%)$ & $0(0)$ & $0(0)$ & \\
Epididymitis, $\mathrm{n}(\%)$ & $0(0)$ & $19(6.5)$ & 0.386 \\
\hline Voiding symptom, $\mathrm{n}(\%)$ & $\mathrm{I}(14.2)$ & $9(3.1)$ & $0.999<$ \\
\hline Urinary retention, $\mathrm{n}(\%)$ & $0(0)$ & $39(13.3)$ & 0.06 \\
\hline Macrohematuria, $\mathrm{n}(\%)$ & $3(42.9)$ & $6(2)$ & $0.999<$ \\
\hline Others, $\mathrm{n}(\%)$ & $0(0)$ & & \\
\hline
\end{tabular}

patients) were diagnosed with fUTI in the negative urine culture group $(p>0.999)$. Statistical analysis revealed that non-infectious complications, such as voiding symptoms ( $14.2 \%$ vs $6.5 \%, \mathrm{p}=0.386)$, urinary retention $(0 \%$ vs $3.1 \%$, $\mathrm{p}>0.999)$, and macrohematuria ( $42.9 \%$ vs $13.3 \%, \mathrm{p}=0.06$ ), were not significantly different between the two groups.

Table 4 shows the association between pre-biopsy urine culture and urine culture upon fUTI diagnosis, considering the time and type of infection, in patients with post-biopsy fUTI. There were four patients with prostatitis and one patient with pyelonephritis. Among these cases, $40 \%$ ( 2 patients) had a positive urine culture at the time of fUTI. All patients with infection were treated with piperacillin-tazobactam with no fatal adverse event.

\section{Discussion}

Of the 300 patients selected in the study, only seven patients $(2.3 \%)$ had a positive urine culture prior to TPPB. In addition, we observed that post-biopsy fUTI was not identified in patients with positive pre-biopsy urine culture but rather in patients with negative urine culture results. This study can be considered a study of asymptomatic patients because patients with indwelling urinary catheters were excluded. To the best of our knowledge, this is the first study to investigate the clinical efficacy of performing pre-biopsy urine culture in the development of fUTI following TP-PB.

The prevalence of prostate cancer has increased partly due to the increased availability of PSA for diagnosis. PB is still considered the gold standard for the diagnosis of

Table 4 The Association Between Pre-Biopsy Urine Culture and Urine Culture Upon Febrile Urinary Tract Infection in Patients with Infection Following Transperineal Prostate Biopsy

\begin{tabular}{|c|c|c|c|c|c|c|c|c|}
\hline Age & $\begin{array}{l}\text { Prostate } \\
\text { Volume }\end{array}$ & $\begin{array}{l}\text { Number } \\
\text { of Biopsy } \\
\text { Cores }\end{array}$ & $\begin{array}{l}\text { Diabetes } \\
\text { Mellitus }\end{array}$ & Immunosuppression & $\begin{array}{l}\text { Urine } \\
\text { Culture } \\
\text { at } \\
\text { Prebiopsy }\end{array}$ & $\begin{array}{l}\text { Urine Culture } \\
\text { at Infection }\end{array}$ & $\begin{array}{l}\text { Type of } \\
\text { Post-Biopsy } \\
\text { Infection }\end{array}$ & $\begin{array}{l}\text { Date of } \\
\text { Infection } \\
\text { Onset (Days } \\
\text { After Biopsy) }\end{array}$ \\
\hline 69 & 41 & 14 & No & No & No growth & Escherichia coli & Prostatitis & 19 \\
\hline 69 & 18 & 20 & No & Yes & No growth & No growth & Prostatitis & I \\
\hline 74 & 70 & 15 & No & No & No growth & No growth & Prostatitis & I \\
\hline 65 & 48 & 14 & No & No & No growth & $\begin{array}{l}\text { Acinetobacter } \\
\text { baumannii / } \\
\text { Staphylococcus } \\
\text { epidermidis }\end{array}$ & Prostatitis & 7 \\
\hline 79 & 63 & 14 & No & No & No growth & No growth & Pyelonephritis & 5 \\
\hline
\end{tabular}


prostate cancer. Although TR-PB is widely performed worldwide, the increasing antibiotic resistance of the rectal flora is associated with the increasing incidence of severe post-biopsy infections. ${ }^{12,13}$ Although severe infections could also occur in TP-PB, this approach avoids contact with the rectal flora, thereby decreasing the likelihood of infection. In our study, the incidence of post-biopsy infectious complications was observed to be $1.7 \%$ (5 out of 300 patients), which is consistent with previous studies $(0.36-$ $3.82 \%) .^{7-9}$ Since PB is a commonly performed procedure, the safety profile of TP-PB must be further investigated to prevent associated infectious complications.

Although several studies have examined the effectiveness of pre-biopsy rectal swab culture, ${ }^{11,16-18}$ only a few studies have explored the clinical utility of urine culture before TR-PB. ${ }^{14,15}$ Bruyère et al reported that 3.4\% (12 of 353 patients who received transrectal needle biopsy) had positive pre-biopsy urine culture $\left(>1 \times 10^{5} \mathrm{CFU} / \mathrm{mL}\right) 48-72$ $\mathrm{h}$ before $\mathrm{PB}$, and only $1.1 \%$ of patients with negative prebiopsy urine culture developed infectious complications following $\mathrm{PB}$. In their study, all patients received ofloxacin $200 \mathrm{mg} \mathrm{2-6} \mathrm{h}$ before PB. ${ }^{14}$ Qi et al have also shown that $4 \%$ (6 of 150 patients who underwent prostate needle biopsy) had evidence of bacteriuria $\left(>1 \times 10^{5} \mathrm{CFU} / \mathrm{mL}\right)$ in urine cultures 14 days before PB. Among these, no patient developed infectious complications following PB. In their study, all patients received prophylactic ciprofloxacin $500 \mathrm{mg}$ the night before and the morning of the biopsy. ${ }^{15}$ Both studies described above have suggested that routine urine culture before planned $\mathrm{PB}$ in asymptomatic patients was unnecessary. Although the cause of infectious complications of TR-PB could be bacteria found in either feces or urine, the results from these studies may be attributed to the more significant influence of the rectal flora on postbiopsy infection than that of urine.

However, infectious complications of TP-PB may be caused by bacteria in the perineal skin or urine. To date, no report has examined the utility of urine culture before TPPB to assess the likelihood of infectious complications. In our study, the incidence of positive urine culture before TP-PB was only $2.3 \%$. Among these patients, no infectious complication was observed. Furthermore, no significant difference was observed between the positive and negative pre-biopsy urine culture groups for background characteristics, such as age, prostate volume, number of biopsies, as well as a history of biopsy, diabetes mellitus, and use of immunosuppressive drugs. Our results suggest that routine pre-biopsy urine culture is unnecessary for treating post-biopsy fUTI in asymptomatic patients.

Another key finding from this study is that a single 1 $\mathrm{g}$ dose of intravenous cefazolin may be sufficient prophylactic antibiotics for fUTI following TP-PB. Some reports have indicated that quinolone-based antibiotics are effective for prophylaxis. ${ }^{19,20}$ However, the development of resistance to quinolone-based antibiotics is an alarming issue, ${ }^{6}$ and the routine use of broad-spectrum antimicrobials, such as quinolones, for prophylaxis remains debatable. However, further prospective randomized controlled trials are necessary to assess the efficacy of cefazolin for prophylaxis.

Several limitations of this study must be acknowledged before interpreting our findings. First, this study was based on a limited population at a single institution; the lack of statistical significance in the analysis may be attributed to a low statistical power due to the small sample size. Furthermore, multivariate analysis for the adjustment for potential confounders was challenging because of the extremely low incidence of post-biopsy infectious complications. Second, the retrospective descriptive design was not ideal for attaining study goals. The optimal study design for investigating the benefit of pre-biopsy urine culture would be a randomized controlled trial that compares patients with and without pre-biopsy urine culture. However, because of the low incidence of positive pre-biopsy urine culture and post-biopsy infection, achieving the large sample size required may not be feasible. In addition, the indwelling urethral catheter after biopsy in the current protocol may be a potential confounding factor of post-biopsy fUTI.

Third, due to the retrospective study, the result of urine analysis before the biopsy was lacking. Also, voiding conditions before biopsy, such as post voiding residual or International Prostate Symptom Score, were lacking. These factors may also be potential confounding factors in considering post-biopsy fUTI. Fourth, the urine culture was considered positive if a bacterial density of more than $1 \times 10^{5} \mathrm{CFU} / \mathrm{mL}$ was observed in accordance with previous studies and guidelines. ${ }^{14,15,21}$ Microbiological criteria for the diagnosis of asymptomatic bacteriuria in men have not been adequately validated. This cutoff needs to be validated to see if it is optimal in the current study setting.

Despite these limitations, this study has demonstrated the association between urine culture before TP-PB and post-biopsy fUTI. Our findings indicated that pre-biopsy urine culture was not helpful for the treatment of postbiopsy infection in TP-PB. The uniqueness of this study is a considerable strength. 


\section{Conclusion}

We examined the results of urine culture before TP-PB. In asymptomatic patients, the incidence of positive urine culture was observed to be very low. Positive pre-biopsy cultures were not associated with the development of post-biopsy fUTI.

\section{Abbreviations}

fUTI, febrile urinary tract infection; PB, prostate biopsy; TR-PB, transrectal prostate biopsy; TP-PB, transperineal prostate biopsy; CFU, colony-forming units; PSA, prostate-specific antigen.

\section{Ethics Approval}

All procedures followed were in accordance with the ethical standards of the responsible committee on human experimentation (institutional and national) and with the Helsinki Declaration of 1964 and its later amendments. The study was approved by the Institutional Review Board of Kagawa University (permission number: 2020-125). The need for informed consent was waived given the retrospective nature of the study.

\section{Author Contributions}

All authors made a significant contribution to the work reported, whether that is in the conception, study design, execution, acquisition of data, analysis and interpretation, or in all these areas; took part in drafting, revising or critically reviewing the article; gave final approval of the version to be published; have agreed on the journal to which the article has been submitted; and agree to be accountable for all aspects of the work.

\section{Funding}

This research received no specific grant from any funding agency in the public, commercial, or not-for-profit sectors.

\section{Disclosure}

The authors have no conflicts of interest to declare.

\section{References}

1. Shen PF, Zhu YC, Wei WR, et al. The results of transperineal versus transrectal prostate biopsy: a systematic review and meta-analysis. Asian J Androl. 2012;14(2):310-315. doi:10.1038/aja.2011.130

2. Takenaka A, Hara R, Ishimura T, et al. A prospective randomized comparison of diagnostic efficacy between transperineal and transrectal 12-core prostate biopsy. Prostate Cancer Prostatic Dis. 2008;11 (2):134-138. doi:10.1038/sj.pcan.4500985
3. Kakehi Y, Naito S, Japanese Urological Association. Complication rates of ultrasound-guided prostate biopsy: a nation-wide survey in Japan. Int $J$ Urol. 2008;15(4):319-321. doi:10.1111/j.14422042.2008.02048.x

4. Tohi Y, Kato T, Matsumoto R, et al. The impact of complications after initial prostate biopsy on repeat protocol biopsy acceptance rate. results from the prostate cancer research international: active surveillance JAPAN study. Int J Clin Oncol. 2020;25(12):2107-2114. doi:10.1007/s10147-020-01761-3

5. Hara R, Jo Y, Fujii T, et al. Optimal approach for prostate cancer detection as initial biopsy: prospective randomized study comparing transperineal versus transrectal systematic 12-core biopsy. Urology. 2008;71(2):191-195. doi:10.1016/j.urology.2007.09.029

6. Taylor S, Margolick J, Abughosh Z, et al. Ciprofloxacin resistance in the faecal carriage of patients undergoing transrectal ultrasound guided prostate biopsy. BJU Int. 2013;111(6):946-953. doi:10.1111/ j.1464-410X.2012.11637.x

7. Namekawa T, Fukasawa S, Komaru A, et al. Prospective evaluation of the safety of transrectal ultrasound-guided transperineal prostate biopsy based on adverse events. Int J Clin Oncol. 2015;20 (6):1185-1191. doi:10.1007/s10147-015-0831-6

8. Togo Y, Kubo T, Taoka R, et al. Occurrence of infection following prostate biopsy procedures in Japan Japanese Research Group for Urinary Tract Infection (JRGU)-A multi-center retrospective study. J Infect Chemother. 2014;20(4):232-237. doi:10.1016/j.jiac.2013.10.003

9. Pepe P, Aragona F. Morbidity after transperineal prostate biopsy in 3000 patients undergoing 12 vs 18 vs more than 24 needle cores. Urology. 2013;81(6):1142-1146. doi:10.1016/j.urology.2013.02.019

10. Packer MG, Russo P, Fair WR. Prophylactic antibiotics and Foley catheter use in transperineal needle biopsy of the prostate. $J$ Urol. 1984;131(4):687-689. doi:10.1016/S0022-5347(17)50580-7

11. Taylor AK, Zembower TR, Nadler RB, et al. Targeted antimicrobial prophylaxis using rectal swab cultures in men undergoing transrectal ultrasound guided prostate biopsy is associated with reduced incidence of postoperative infectious complications and cost of care. J Urol. 2012;187(4):1275-1279. doi:10.1016/j.juro.2011.11.115

12. Cuevas O, Oteo J, Lázaro E, et al. Significant ecological impact on the progression of fluoroquinolone resistance in Escherichia coli with increased community use of moxifloxacin, levofloxacin and amoxicillin/clavulanic acid. J Antimicrob Chemother. 2011;66(3):664-669. doi:10.1093/jac/dkq471

13. Loeb S, Carter HB, Berndt SI, Ricker W, Schaeffer EM. Complications after prostate biopsy: data from SEER-Medicare. J Urol. 2011;186(5):1830-1834. doi:10.1016/j.juro.2011.06.057

14. Bruyère $\mathrm{F}$, d'Arcier BF, Boutin JM, Haillot $O$. Is urine culture routinely necessary before prostate biopsy? Prostate Cancer Prostatic Dis. 2010;13(3):260-262. doi:10.1038/pcan.2010.8

15. Qi DZ, Lehman K, Dewan K, Kirimanjeswara G, Raman JD. Preoperative urine culture is unnecessary in asymptomatic men prior to prostate needle biopsy. Int Urol Nephrol. 2018;50(1):21-24. doi:10.1007/s11255-017-1752-2

16. Duplessis CA, Bavaro M, Simons MP, et al. Rectal cultures before transrectal ultrasound-guided prostate biopsy reduce post-prostatic biopsy infection rates. Urology. 2012;79(3):556-561. doi:10.1016/j. urology.2011.09.057

17. Hiyama Y, Takahashi S, Uehara T, et al. Selective culture of Escherichia coli to prevent infective complications of transrectal ultrasound-guided prostate biopsy: clinical efficacy and analysis of characteristics of quinolone-resistant Escherichia coli. Int $J$ Urol. 2019;26(6):655-660. doi:10.1111/iju.13960

18. Würnschimmel C, Busto Martin LB, Leyh-Bannurah SR, et al. Rectal swabs for detecting multidrug resistant bacteria prior to transrectal prostate fusion biopsy: a prospective evaluation of risk factor screening and microbiologic findings. Urology. 2020;136:127-132. doi:10.1016/j.urology.2019.10.008 
19. Pepe P, Aragona F. Prostate biopsy: results and advantages of the transperineal approach-twenty-year experience of a single center. World J Urol. 2014;32(2):373-377. doi:10.1007/s00345-013-1108-1

20. Symons JL, Huo A, Yuen CL, et al. Outcomes of transperineal template-guided prostate biopsy in 409 patients. BJU Int. 2013;112 (5):585-593. doi:10.1111/j.1464-410X.2012.11657.x
21. Nicolle LE, Bradley S, Colgan R, et al. Infectious Diseases Society of America guidelines for the diagnosis and treatment of asymptomatic bacteriuria in adults. Clin Infect Dis. 2005;40(5):643-654. doi:10.1086/427507

\section{Publish your work in this journal}

Research and Reports in Urology is an international, peer-reviewed, open access journal publishing original research, reports, editorials, reviews and commentaries on all aspects of adult and pediatric urology in the clinic and laboratory including the following topics: Pathology, pathophysiology of urological disease; Investigation and treatment of urological disease; Pharmacology of drugs used for the treatment of urological disease. The manuscript management system is completely online and includes a very quick and fair peer-review system, which is all easy to use. Visit http://www.dovepress.com/ testimonials.php to read real quotes from published authors. 